\title{
Fatigue analysis of cracks at the rib-to-deck welded joint of orthotropic steel deck accounting for the influence of compressive stress in cyclic loading
}

\author{
Ming Deng ${ }^{1,2, a}$, Qing $\mathrm{Du}^{3}$ and Ningfei Huo ${ }^{3}$ \\ ${ }^{1}$ School of Civil Engineering \& Architecture, Changsha University of Science\& Technology, \\ Changsha Hunan, 410114, China \\ ${ }^{2}$ Tianjin Transportation Research Institute, Tianjin 300300, China \\ ${ }^{3}$ Department of Mechanics, Tianjin University, Tianjin 300354, China
}

adengming_979@126.com

Keywords: Orthotropic steel bridge deck, Rib-to-deck welded joint, Fatigue analysis, Cyclic loading with negative stress ratio, Influence of compressive stress, Reverse plastic zone at crack tip

\begin{abstract}
This paper presents the fatigue analysis of surface cracks at the rib-to-deck welded joint of orthotropic steel deck, in which the influence of the compressive stress in the cyclic loading on the crack growth is taken into account. The stress intensity factors along the surface crack front at the rib-to-deck welded joint are evaluated efficiently by Schwartz-Neuman alternating method based on a 3D local model of the rib-to-deck welded joint containing the crack. The influence of the compressive stress in cyclic loading on the fatigue crack growth is taken into account by a reverse plastic zone model at the crack tip of a 2D fracture computational mode. The results show that the compressive stress in the cyclic loading significantly influences the growth of the fatigue cracks at the rib-to-deck welded joints. The present study also indicates that the rib-to-deck welded joint of the orthotropic steel deck meets the fatigue design requirement when the thickness of the deck plate is $14 \mathrm{~mm}$.
\end{abstract}

\section{Introduction}

The orthotropic steel bridge deck is made up of longitudinal, transverse stiffeners, bridge plates and pavement. Because of the advantages of light weight, big ultimate bearing capacity, easy to assemble connection, short construction period, beautiful appearance and so on, are widely used in large span bridges. The initial defects at the welded joints of the orthotropic steel decks will undergo fatigue failure under the action of live load of moving trucks on the bridge decks $[1,2]$. Therefore, the fatigue life evaluation is very important for the safety of orthotropic steel decks $[2,3]$. The localized transverse stresses at the rib-to-deck joints of orthotropic steel decks are the most sensitive parts to the live loads of truck traffics, and the cracks at the rib-to-deck welded joints are the defects most prone to fatigue failure under the random loading of moving trucks [4].

Some scholars analyzed the impact of compressive stress on the crack propagation from the perspective of elastic-plastic mechanics. Sha [4] pointed out that under the condition of the negative stress ratio in cycle loading, the reverse plastic zone generated at last cycle period can be more serious sharpening and passivity in the crack tip, then they put forward the crack propagation formula which considers the influence of the maximum compressive stress.

The objective of the present work is to evaluate the fatigue life of the surface cracks at the rib-to-deck welded joint of orthotropic steel deck, where the fatigue cracks are under the action of the cyclic loading with negative stress ratio. The fatigue analysis is based on the fatigue crack growth rate with the consideration of the influence of compressive stress in the cyclic loading. A five-step approach was employed to analyze the fatigue crack growth of the rib-to-deck welded joint of orthotropic steel deck. The first step is the stress analysis of the rib-to-deck welded joint, which is similar to that used in the common two-step approach of the fracture mechanics-based fatigue analysis of steel bridge decks [1]. The second step is the calculation of the stress intensity 
factors along the surface crack fronts at the rib-to-deck welded joint using Schwartz-Neuman alternating method based on a 3D local model. The third step is the evaluation of the influence of the compressive stress in cyclic loading on the fatigue crack growth using a reverse plastic zone model at crack tip. The fourth step is to calculate the fatigue crack growth rate of the surface cracks at the rib-to-deck welded joint by substituting the stress intensity factors given in the second step to the crack growth formula obtained in the third step. The last step is the evaluation of the fatigue life of the surface cracks of the rib-to-deck welded joint of orthotropic steel deck, where the simplification of the cyclic loading with constant stress amplitude is used.

\section{The Transverse Stress at the Rib-to-Deck Welded Joint}

A schematic of the U-rib, floor beams and deck plate of an orthotropic steel deck is illustrated in Fig. 1a. It has been shown that the cracks at the rib-to-deck welded joints of orthotropic steel decks are the most sensitive cracks prone to fatigue crack growth under the cyclic loadings induced by moving trucks on the bridge decks $[1,3]$. The locations of the surface crack of the rib-to-deck welded joint of orthotropic steel deck are depicted in Fig. 1b. The computational model of the stress analysis for the stress fatigue assessment of the orthotropic steel bridge deck is composed of five longitudinal U-ribs and four floor beams. The thickness of the deck plate is $14 \mathrm{~mm}$, and the span between the two neighboring floor beams is $3 \mathrm{~m}$. The dimensions of the U-rib are $300 \mathrm{~mm}$ and 200 $\mathrm{mm}$ at its top and bottom respectively, and the height is $280 \mathrm{~mm}$. The thickness of the U-rib is 8 $\mathrm{mm}$, and the spacing of the U-ribs is $600 \mathrm{~mm}$. The height of the floor beam is $700 \mathrm{~mm}$, and the thickness is $12 \mathrm{~mm}$. The material used in the orthotropic steel decks is Q345q steel. The elastic modulus of the material is $206 \mathrm{GPa}$ and the Poisson's ratio is 0.3 .

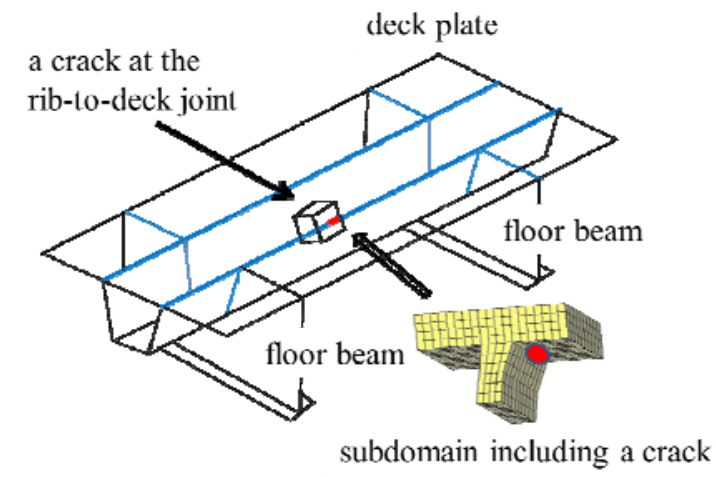

(a) schematic of U-rib and a 3D local crack model

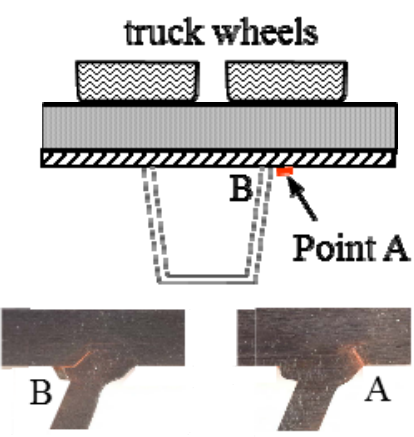

(b) cracks at rib-to-deck welded joint

Fig. 1 The schematic of the U-rib of orthotropic steel deck, the cracks at rib-to-deck welded joint and a 3D local model for a crack at the toe of rib-to-deck welded joint

ANSYS was used for the stress analysis of the fatigue evaluation, in which the contribution of the pavement to the bending stiffness is neglected. The deck plate, U-ribs and floor beams are all modeled by shell element Shell181 in ANSYS. The total number of the shell elements is 76300 . The displacement boundary conditions [3] are used for the computational model of the stress analysis. Shi et al [3] pointed out that the loads of the two wheel-axles of heavy trucks have to be considered in order to accurately calculate the tensile transverse stresses at the rib-to-deck welded joints of orthotropic steel decks when the distance between two neighboring wheel-axles is smaller than the distance between two neighboring floor beams. Therefore, the dual wheel loads of double wheel axes of the standard fatigue load vehicle are used for the analysis of stresses at the rib-to-deck welded joints of orthotropic steel deck. The load of the dual wheels is $70 \mathrm{kN}$, and the distance between two neighboring wheel axes is $1.4 \mathrm{~m}$. The over-rib loading of the truck wheels shown in Fig. 1b is adopted in this work. When two neighboring axes are located around the mid-span of two floor beams, the resulting influence lines of the transverse stress at the rib-to-deck welded joints between two floor beams are plotted in Fig. 2. One can see that the tensile stress 
resulting from the wheel loads of double wheel axes is higher than that given by the load of single wheel axe. Since the fatigue crack growth is more sensitive to the tensile stress in the cyclic loading, the tensile stress shown in Fig. 2 is the main driving force of the crack growth at rib-to-deck welded joints [3].

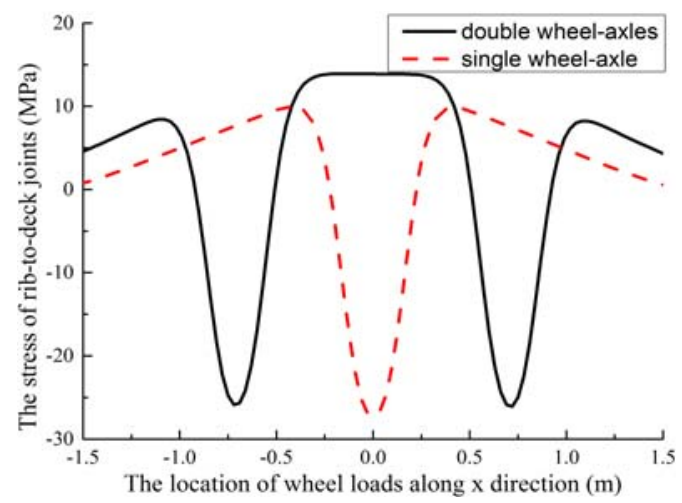

Fig. 2 The influence lines of the transverse stress at rib-to-deck welded joint between two floor beams.

The different location of the wheels along the span of two neighboring floor beams results in different influence lines of the transverse stress at the rib-to-deck welded joints. It can be seen from Fig. 2 that the rib-to-deck welded joint is under the action of the cyclic loading with negative stress ratio induced by the moving truck on the bridge. Fig. $1 \mathrm{~b}$ also indicates that the compressive stress is the dominant part in the cyclic loading at the rib-to-deck welded joints.

\section{Fracture Mechanics Analysis of Surface Cracks at the Rib-to-Deck Welded Joint}

The Schwartz-Neuman alternating method [5] is an efficient model for the 3D fracture mechanics analysis of the rib-to-deck welded joints of orthotropic steel deck, in which a local 3D model of the rib-to-deck welded joint containing a crack as shown in Fig. 1a is used for the computational model of the evaluation of the stress intensity factors (SIF).

The crack in the Schwartz-Neuman alternating method is characterized by a boundary element model. The semi-elliptical surface crack at both the weld toe and the weld root, that are corresponding to Point A and Point B depicted in Fig. 1a respectively, are considered. The dimensions of the semi-elliptical surface crack are of $3 \mathrm{~mm}$ for the semi-long axis and $2 \mathrm{~mm}$ for the semi-short axis. The maximum tensile stress shown in Fig. $1 \mathrm{~b}$ is used as the stress values on the local 3D model containing a surface crack for the fracture mechanics analysis in this paper. The distribution of SIFs along the crack fronts are illustrated in Fig. 3. It can be seen from the SIF curves in Fig. 2 the crack at the weld toe is more prone to fatigue failure than that at the weld root.

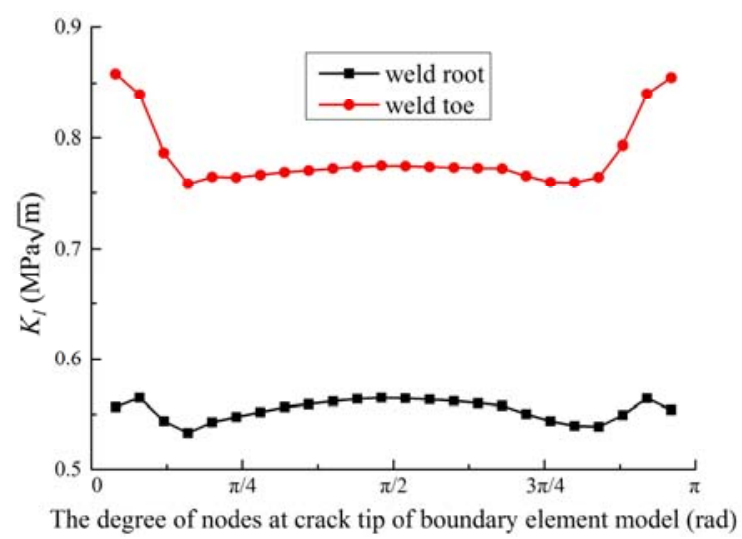

Fig. 3 The distributions of SIFs along the elliptical surface crack fronts at, respectively, the weld root and weld toe of rib-to-deck welded joint. 


\section{Influence of Compressive Stress in Cyclic Loading on Fatigue Crack Growth}

As shown in Fig. 1b, the rib-to-deck welded joint of orthotropic steel deck is under the action of cyclic loading with negative stress ratio. The influence of the compressive stress in the cyclic loading on the crack growth has to be taken into account. However, now it is still an open question on how to characterize the effect of compressive stress in the cyclic loading on the crack growth. Based on the reverse plastic zone model, Sha [4] proposed to use the SIF $K_{\max }$ corresponding to the maximum tensile stress in the cyclic loading and the maximum compressive stress $\sigma_{\text {maxcom }}$ (in terms of the absolute value) in the cyclic loading to evaluate the fatigue crack growth under the action of cyclic loading with negative stress ratio. The crack growth model is of the form

$$
\frac{\mathrm{d} a}{\mathrm{~d} N}=C\left(1-\gamma \sigma_{\max c o m}\right)^{\beta}\left(K_{\max }\right)^{m}
$$

in which $C, m, \beta$ and $\gamma$ are the material constants. $C$ and $m$ can be obtained from the existing experimental data used for Paris law, and $C=9.03 \times 10-11, m=3.3$ [5] are used in this work. $\beta$ can be determined from two groups of different stress ratios $R_{1}$ and $R_{2}$ used for Paris law. $\beta=0.68$ is taken here. $\gamma$ can be measured from the experiments for the given material or evaluated numerically from the size of the reverse plastic zone of the given material by the following equation [4]

$$
R_{r p \max }=\left(1-\gamma \sigma_{\max c o m}\right) \frac{K_{\max }^{2}}{4 \pi \sigma_{y s}^{2}}
$$

where, $R_{r p \max }$ is the maximum size of reverse plastic zone and $\sigma_{y s}$ is the yielding stress of the material under consideration. The material constants $\gamma=0.14 \mathrm{MPa}^{-1}$ for Q345q steel, in which the plane strain condition was assumed. Then it follows Eq. (1) that the crack growth rate of Q345q steel with the effect of the compressive stress in cyclic loading is given by

$$
\frac{\mathrm{d} a}{\mathrm{~d} N}=9.03 \times 10^{-11} \times\left(1-0.14 \sigma_{\max c o m}\right)^{0.68}\left(K_{\max }\right)^{3.3}
$$

\section{Growth Rate of Surface Crack at the Rib-to-Deck Welded with the Effect of Compressive Stress in Cyclic Loading}

By substituting the $\sigma_{\text {maxcom }}$ corresponding to the double wheel-axes shown in Fig. 2 into Eq. (3), the crack growth rates along the elliptical surface crack corresponding to the SIFs shown in Fig. 3 are plotted in Fig. 4. When the influence of the compressive stress in the cyclic loading is taken into account in the manner modeled by Eq. (3), the crack growth rates is about 2.5 times of that given by Paris law which only takes the tensile stress into account. Therefore, the influence of the compressive stress in the cyclic loading on the growth of the fatigue cracks at the rib-to-deck welded joints is very significant.

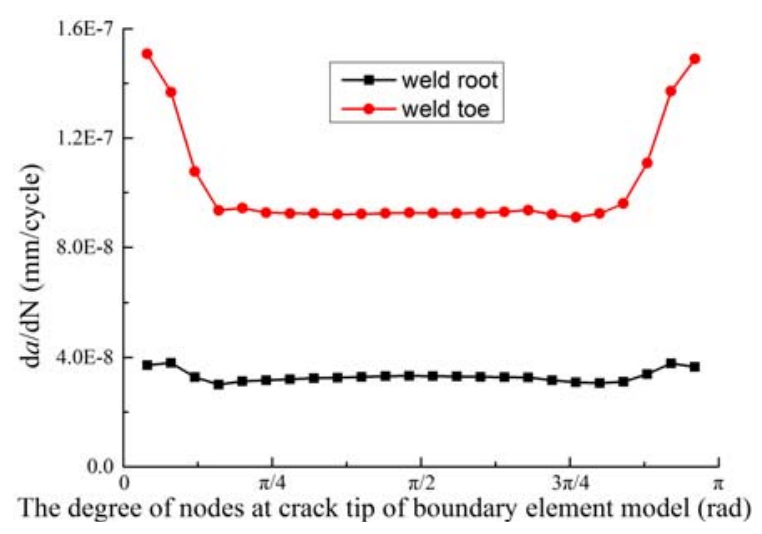

Fig. 4 The crack growth rates along the elliptical surface crack tips at the weld root and weld toe of rib-to-deck welded joint 


\section{Fatigue Analysis of Surface Cracks at the Rib-to-Deck Welded Joint Accounting for the Effect of Compressive Stress in Cyclic Loading}

The stress spectrum at the rib-to-deck welded joints of orthotropic steel bridge deck is very complicated as the real loading conditions induced by the moving trucks on the bridge is complex, as the magnitudes of truck loads are varying and the contact areas of the truck wheels with respect to the ribs are random. The stress analysis for the fatigue evaluation of rib-to-deck welded joints presented in Section 1 is based on the worst loading case. Consequently, a conservative fatigue evaluation can be performed by using the constant stress range given by the over-rib loading of the dual wheels of the trucks with load of $70 \mathrm{kN}$ considered in Section. It can be seen from Fig. 4 that it takes more than two million cycles for the crack propagation of $1 \mathrm{~mm}$ along the deck plate thickness. The fatigue life of orthotropic steel bridge decks is usually two million cycles of heavy truck loading, therefore, the rib-to-deck welded joints of the orthotropic steel deck with the deck plate of $14 \mathrm{~mm}$ is strong enough.

\section{Conclusions}

The rib-to-deck welded joint of orthotropic steel deck is under the action of the cyclic loading with the tensile-compressive stress where the compressive stress is the dominant part. As a result, the fatigue analysis of the rib-to-deck welded joint of orthotropic steel deck is very challenging. The fatigue evaluation of the rib-to-deck welded joint of orthotropic steel deck with the effect of the compressive stress in the cyclic loading is presented in this paper. The influence of the compressive stress in cyclic loading on the fatigue crack growth is characterized by a reverse plastic zone model at the crack tip with a $2 \mathrm{D}$ fracture computational mode. The results show that the crack growth rates considering the influence of the compressive stress in the cyclic loading is about 2.5 times of that given by Paris law which only takes the tensile stress into account. Therefore, the influence of the compressive stress in the cyclic loading on the growth of the fatigue cracks at the rib-to-deck welded joints has to be taken into account. A preliminary fatigue analysis shows that the rib-to-deck welded joint of the orthotropic steel deck meets the fatigue design requirement when the thickness of the steel bridge deck is $14 \mathrm{~mm}$.

\section{Acknowledgement}

In this paper, the research was sponsored by Project of Tianjin Urban \& Rural Construction Municipal Commission of Science and Technology (Project No. 201510) and Project of Tianjin Municipal Transportation Commission of Science and Technology (Project No. 2016A-06).

\section{References}

[1] K. Kiss, K. Dunai. Fracture mechanics based fatigue analysis of steel bridge decks by two-level cracked models. Comput. Struct. 80 (2002) 2321-2331.

[2] G. Zhang, G. Shi, X. Wang. Evaluation of the approaches for fatigue life prediction of welded joints of orthotropic steel decks and some suggestions. Chinese J. Solid Mech. S1 (2013) 216-223.

[3] G. Shi, X. Li, G. Zhang. Evaluation of stress intensity factor range in the prediction of fatigue crack growth at rib-to-deck welded joints of orthotropic steel decks. Adv. Mater. Res. 671-674 (2013) 969-973.

[4] Y. Sha, J. Zhang. Study on the effect of compression loading on fatigue crack growth of aluminium under tension-compression cycle. Eng. Mech. 29(10) (2012) 327-334.

[5] Z. D. Han, S. N. Atluri. SGBEM (for cracked local subdomain)-FEM (for uncracked global structure) alternating method for analyzing 3D surface cracks and their fatigue-growth. CMES: Comput. Mod. Eng. Sci. 6 (2012) 699-716. 
[6] L. Zong, G. Shi et al. Experimental study on fatigue crack growth rate of Q345qD bridge steel. J. Railway Sci. Eng. 36(3) (2015) 37-44.

[7] Q. Du, G. Shi. Crack growth analysis of the rib-to-deck welded joints of orthotropic steel deck under cyclic loading with negative stress ratio, (submitted). 